\title{
Treatment of Cervical Cancer with Ointment After Radiotherapy and Chemotherapy
}

\author{
Wan Peiyu, Cai Jing* \\ Shaanxi University of Traditional Chinese Medicine, Xianyang, Shaanxi, 712000, China.
}

\begin{abstract}
How to cite this paper: Wan Peiyu, Cai Jing. (2020) Treatment of Cervical Cancer with Ointment After Radiotherapy and Chemotherapy. International Journal of Clinical and Experimental Medicine Research, 4(3), 69-72. DOI: 10.26855/ijcemr.2020.07.007
\end{abstract}

Received: May 12, 2020

Accepted: June 10, 2020

Published: July 10, 2020

*Corresponding author: Cai Jing, MD, Chief Medical Officer, Teacher, Master tutor, Shaanxi University of Traditional Chinese Medicine, Xianyang, Shaanxi, 712000, China. Email: 1308843516@qq.com

\begin{abstract}
Cervical cancer is one of the most common malignant tumors in gynecology. With the incidence rate becoming younger in recent years, although advanced screening methods and preventive vaccines are used, the treatment plan is still limited to surgery, radiotherapy and chemotherapy, and all have advantages and disadvantages. After radiotherapy and chemotherapy, cervical cancer belongs to the category of "deficiency of qi and blood", "deficiency of labor" and "deficiency damage" of traditional Chinese medicine. Under the guidance of TCM theory, we developed Ginseng Poria cocos Ejiao ointment with the method of "invigorating qi and nourishing blood and nourishing yang and solid foundation". This article mainly describes the cervical cancer after the release. In order to provide new ideas for the future clinical diagnosis and treatment, know the etiology and pathogenesis of post-treatment diseases.
\end{abstract}

\section{Keywords}

Poria cocos gelatin cream, cervical cancer, postoperative chemoradiotherapy

Cervical cancer is the most common gynecological malignant tumor, it is a serious threat to women's physical and mental health, and younger and more obvious [1]. Comprehensive measures such as radiotherapy and chemotherapy have become an effective method to improve the long-term survival rate of patients with cervical cancer after operation, but the toxic side effects caused by some patients are difficult to tolerate, which directly affects the success or failure of cervical cancer treatment. In recent years, a large number of studies have reported that Chinese medicine has a significant detoxification and synergistic effect on chemotherapy in patients after tumor surgery [2]. As a common dosage form of traditional Chinese medicine (TCM) for the treatment of chronic diseases, the prescription can best reflect the characteristics of TCM treatment and has a unique advantage in the treatment of tumors [3-4]. Ointment the principle of regulating yin and yang qi and blood can improve physique, replenish deficiency and win, and correct and exorcise evil spirits. "Ointment Fang Daquan" has long been discussed: "Ointment Fang, cover the boil into fat liquid, and so nutrition of the internal organs of the boring weak." In order to provide some reference for the clinical practice and research on the treatment of cervical cancer after radiotherapy and chemotherapy, the patients with cervical cancer had deficiency of qi and accumulation of evil toxin.

\section{Pay attention to pathogenesis and syndrome differentiation}

Bone marrow suppression after radiotherapy and chemotherapy after cervical cancer can be classified as "deficiency labor" and "blood deficiency". The endogeny of cancer toxin is caused by deficiency of qi, internal invasion of external evil dampness toxin and accumulation of phlegm and blood stasis [6]. As described in the Book of Saint-Ji, "The tumour is just for righteousness, but it is left behind.” Qi and blood epidemic without losing its part, then the body is peaceful, no or residual burden. Long depression accumulation congestion, then by the empty cast 
gap, disease from the birth. The course of cervical cancer is prolonged, chemotherapy is more injured qi, loss of blood, Make liver, spleen and kidney and other viscera qi and blood imbalance, qi and blood biochemical deficiency, water valley essence lost in the transport, essence is not filled and pathogenic.

\subsection{Where evil is gathered, its spirit will be empty}

Chinese medicine believes that chemotherapeutic drugs are heat-induced yang poison, which is external evil, which is originally poison, can treat cancer poison by poison attack poison, evil poison into body, qi and fight against it, good and evil fight, poison evil is too prosperous, qi is easy to deficiency, disturb viscera qi machine, damage spleen and kidney, spleen deficiency causes qi damage, qi and blood injury, yin and yang disorder is the main pathogenesis of the disease. Remaining cancer is poison, chemotherapy drugs are also poison, are tangible body, accumulate human body and consume Yin Qi, evil Qi damage, body qi deficiency, Yang Qi partial decline, Yin essence blood fluid deficiency, cannot honor the body. Yang deficiency gasification is unfavorable, the body water fluid and Yin essence metabolism disorder, condensed into phlegm, phlegm Qi blocking machine, and the blood flow is not smooth, choroid stasis, phlegm and blood stasis for a long time and brewing toxic swelling. "Internal Classic" has long been recorded: "the accumulation of evil qi stagnation, and phlegm drink, blood fetish cementation, accumulated years, fetish and internal storage, evil blood is born.” It has been clarified that the accumulation of evil qi in the body to generate phlegm and blood stasis, long-lasting and evil blood. Zhang Jingyue also said: "blood-gas gathering, cannot be dissolved, its poison such as Gu, blood stasis stagnation.” Say its yang qi is insufficient, blood stasis is not scattered stagnation and become ill. After radiotherapy and chemotherapy of cervical cancer, although the cancer poison is gone, and the remaining poison, drug poison love and body, injury to the body Yin essence, phlegm and blood stasis poison, long time do not cross the body, no. With "Yin essence" forming too much, make Yang Qi is not enough, "Yang Qi, Yin into form”, "Yang deficiency is Yin Sheng”, "no Yang is Yin without change". Sun Yaoxian and others said that the essence of cancer disease is "garbage Yin essence", did not get normal Yang Qi warm steam, and accumulate the body. "Medical sect must read" on the accumulation of the successful, "Qi is not enough, and then the evil spirit." "Accumulation, is Yin Qi is also", so cancer after radiotherapy and chemotherapy with " poison” evil accumulation, love Yin damage Yang and disease.

\subsection{A good spirit exists, but evil does not}

Zhengqi is a Qi of the Yuan Yang, depending on the essence of blood body fluid filling and gasification. Zhengqi Sheng, then can win evil, Zheng Sheng is not the body disease. After radiotherapy and chemotherapy after cervical surgery, it is easy to injure qi, consume essence and blood, make spleen and kidney function more and more dysfunctional, water valley fine transport unfavorable, qi and blood biochemical source, essence cannot be filled, and qi and blood loss, a series of bone marrow suppression and anemia signs. "The middle coke receives the qi to take the juice, changes but the red is the blood", the blood is the middle coke spleen and stomach qi and metaplasia, then nourishes the whole body. "Medical Zong Jin Jian" records: "One has this body. It is necessary to be in the stomach, the valley into the stomach, sprinkle Chen in the six-Fu and Qi to, and adjust in the five viscera and blood born", and can be a person for a living. "Kidney contains essence, the essence of the blood is also", so the essence of the human body is mainly acquired by the spleen and stomach of the water valley refined and "heart red" and blood. Therefore, "spleen and kidney" plays an important role in blood production.

"The essence" said: "Kidney is the essence of vitality, the root of the generation, in order to maintain the way is always.” Explicitly discuss the root of Qi in the kidney, kidney essence filled, active metaplasia, Qi filled, strong body. Cervical cancer is also a disease of women." Women are born with liver "and" mainly with blood. Blood relies on Qi line, blood relies on gasification, Qi line is blood line, Qi, Qi Wang is blood. Qi, Yang also. Blood, Yin also. One of the cancer syndromes, non-yang cannot send its poison, non-yin cannot turn its poison, so must get its Yin and Yang rendezvous, Qi and blood is also smooth. After radiotherapy and chemotherapy, Qi and blood were out of harmony and Yin and Yang were out of balance. Women to blood-based, the responsibility of the spleen and kidney, spleen and kidney damage, essence and blood cannot be metaplastic, qi loss in charge and disease.

\section{Characteristics and Application of Ointment in Cancer Therapy}

Ointment, first found in the Shanhai Classic, "good words and good taste are slippery as ointment” [6]. Qin Bo did not point out in "Ointment Fang Daquan" [7] that the ointment is by: "suffering medicine juice or fat liquid and so nutrition of the internal organs of the boring weak also", out of its nourishing the five viscera qi, deficiency weak effect. And the whole prescription "is not a simple tonic, but contains the meaning of saving bias but dis- 
ease”, compared with other dosage forms, there are synergistic and slow toxicity, regulating immune effect [8-10]. This out of the ointment is not only nourishing strong, but also has the function of balancing yin and yang qi and blood, so that the human body to reach the state of yin and yang secret, in line with "yin and yang Sheng and tune it, to flat for a period" the principle of treatment, especially in patients with chronic prolonged postoperative radiotherapy and chemotherapy of tumors [11].

I believe that the use of ointment in the treatment of cervical cancer after radiotherapy and chemotherapy of the characteristics of the drug is that it is to strengthen the spirit, can protect the spleen and stomach qi, increases the efficiency of detoxification. Especially to emphasize "regulating qi” and "regulating spleen”, to support yang to suppress yin, nourish spleen and kidney as the law, to strengthen the protection of qi, metaplasia of essence and blood, in order to dispel evil poison qi.

\subsection{Fuyang Guji}

"Lingshu hundred diseases first born" said: "the beginning of the product, cold is born, syncope is also a product," and said its "not warm qi, coagulation and not scattered," it is clear that "product" needs to be warm and walk, "blood gas, like warm and evil cold", cancer after the release of chemotherapy after the "residual poison" for accumulation and not, phlegm and blood stasis, cross disease. "Synopsis of the Golden Chamber" discussed: "disease phlegm drink, when the warm yang harmony", has proposed the treatment of tangible evil, warming yang qi as its main treatment. By warming Yang to supplement Yang Qi, restore the function of "Yang Hua Qi”, so that the tumor "residual poison" diffuse into invisible things. Hu Wenbin et al. [12]. Taking the traditional Chinese medicine of warm and warm yang to raise the yang qi in the body to inhibit the tendency of "yin formation" and accelerate the diffusion of "useless yin" to prevent and treat bone tumors; Zhao Xizhong [13] and others will nourish spleen and kidney yang qi and inhibit yin formation by promoting the function of "yang qi" to treat malignant tumors; Miao Xidi [14] is the pathogenesis of treating tumors. And Zhang Yongjie et al. [15] use the Wenyang method to treat the advanced non-small cell lung cancer chemotherapy, gives the Ma Fu Yiyang decoction, exacerbates the aconite Fu Yang's work, its clinical curative effect is very good. "Yang master Yin from, Yang Sheng Yin long, Yang kill Yin Zang”, so treatment "Yu Poison Yin”, to warm Yang Qi method. Zhu Wei chrysanthemum in "typhoid quality is difficult" said: "yang does not suffer much, it is in the secret, is its treatment, when the first Chongyang use; yang decline a point, then disease into a point; is flourishing a point, but a point of disease, this inevitable reason is also, get yang life, loss of yang died”, more clarify the importance of taking care of Yang Qi and strengthening the spirit of exorcism [16].

\subsection{Invigorating qi and nourishing blood}

"Heaven and earth together Qi, life said people”, people are the products of heaven and earth, human life activities are not separated from Qi. Dirty to gas full for the good, Fu to gas for the good, limbs to gas for the use, tendons to gas smooth for the smooth. Cervical cancer patients, tumor accumulation, which is the potential of qi-qi inverse disorder, plus surgery, radiotherapy and chemotherapy and other treatments make the body qi-qi stagnation, qi and blood reverse chaos, and become other diseases [17]. "Drink into the stomach, swim overflow essence, on the spleen, temper scattered essence, on the lung”, "spleen for the stomach of its body fluid", all said the spleen hide essence, transmission of fine work. Kidney contains essence, for the root of Qi, spleen and kidney phase, essence and blood active, Qi and blood Harmony, then the five viscera peace. If the essence of the lack of blood, the body deficiency of qi, cannot drum evil go out.

"Lingshu Wuwei Wuyin” said: “a woman's life, more than Qi, less than blood”, blood is the mother of Qi, Qi is the handsome blood, Qi and blood depend on each other. Therefore, to give full play to the advantages of ointment, to "invigorate qi and nourish blood, strengthen and strengthen the root" as the method, improve adverse reactions, regulate the patient's qi and blood, and strengthen the source of the root, to achieve the purpose of nourishing and dispelling disease.

After many years of clinical experience, the professor of our hospital developed Ginseng Poria cocos Ejiao ointment for the treatment of cervical cancer after radiotherapy and chemotherapy, the clinical effect is very good. In the prescription, we mainly use ginseng to replenish the qi of yang, "replenish the five viscera", "replenish the deficiency of yang qi of spleen and stomach”. Chinese yam tonify spleen, lung, kidney, qi and blood, Lycium barbarum tonify liver and kidney, strengthen the root of vital qi, so as to protect qi, so as to keep evil spirit; Ejiao, longan meat nourishing yin and nourishing blood, while giving Poria cocos, Atractylodes macrocephala, such as invigorating spleen and stomach qi, make qi and blood biochemical active, blood around the body. Also afraid of getting tired of stomach, with Amomum kernel Qi, peach kernel blood, so that the flow of qi and blood smooth 
and orderly; liquorice can detoxify, but also can reconcile the various drugs. The main prescription to warm Yang Qi as the main method, supplemented by nourishing yin and tonifying spleen and kidney qi and blood, strong qi, dispelling the remaining poison.

"Pro-Certificate Guide Medical Cases": "the treatment of the need, with the method of attack should be slow appropriate song, with the method to avoid acerbity avoid staying”, said in the treatment of drug compatibility must be dynamic and dynamic degree, the corresponding. Because of its postoperative, radiotherapy and chemotherapy after the emergence of chronic deficiency of the sign, although the ointment is not as quick as the soup, but can be slow to increase efficiency and detoxification, as Ye Tianshi said: "Wang Dao has no near work, take more self-beneficial". At the same time, clinical treatment also needs to be specific according to the cause of medication, vary from person to person, can achieve clinical effect.

\section{References}

[1] Shi Jin, Liang Di, Li Daojuan, et al. (2017). Analysis of cervical cancer incidence and death status and 40 years death trend in Hebei Province, 2010-2013 [J]. Oncology, 37(11): 1172-1178.

[2] He Xinai, Gao Nana, Ma Xin, et al. (2016). Effect of traditional Chinese medicine on quality of life and side effects of radiotherapy and chemotherapy after breast cancer [J]. Oncology, 6(5): 349-355.

[3] Tao Guoshui, Cha Mingbao, Kong Lingjing, et al. (2014). Analysis on the Key Points and Principles of Prescription for Treating Malignant Tumor [J]. Chinese Medicine Journal, 55(8): 654-656.

[4] Zhou Huamiao, Guo Yong. (2010). Application of ointment in cancer patients [C]. Proceedings of the National Conference on Cancer of Integrated Chinese and Western Medicine, 2010.

[5] Han Fengjuan, Jiang Tingting, Zhang Ming. (2018). Effect of traditional Chinese medicine on cervical cancer and its mechanism [J]. Journal of Tianjin University of Traditional Chinese Medicine, 37(1): 813-817.

[6] Wang Xuqian. (2016). M. of Chinese Medicine China Pharmaceutical Science and Technology Press, 2016.

[7] Qin Bowei. (2013). [M]. Shanghai: Shanghai Science and Technology Literature Publishing House, 2013: 93.

[8] Huang Wenqi, Tang Dongxin, Long Fengxi, et al. (2017). Analysis on the Role of Ointment in Cancer Treatment [J]. Asia Pacific Traditional Medicine, 13(3): 79-81.

[9] Yang Bing, Luo Li, Long Fengxi, et al. (2017). Discussion on the Significance of Plaster in the Prevention and Treatment of Cancer [J]. Chinese Medicine Journal, 32(3): 322-324.

[10] Wang Yisheng, Xia Liming. (2010). Discussion on the Characteristics of TCM Ointment in Treating Malignant Tumor [J]. Journal of Jiangxi University of Traditional Chinese Medicine, 22(5): 7-9.

[11] Discussion on the Application of Zhou Huamiao, Guo Yong and Yifang in Cancer Patients [J].] The Third International Conference of Traditional Chinese Medicine, Integrated Chinese and Western Medicine Oncology and the 12th National Conference on Integrated Chinese and Western Medicine Oncology.

[12] Hu Wenbin, Zhang Hua, Song Min. (2015). [J] Western Chinese Medicine, 8(8): 51-53.

[13] Zhao Xizhong, Yang Xiangdong, Jia Yingtian. (2012). Guiding significance of the theory of "Yang Hua Qi, Yin Cheng” for the treatment of rectal tumor [J]. Modern Journal of Integrated Chinese and Western Medicine, 21(21): 2359-2360.

[14] Miao Xidi, Qin Yong. (2016). "Yang Hua Qi, Yin Formation” and Fuyang Suppressing Yin-Pei-Pei Yuan “in treating tumor”. [J]. Journal of Practical Chinese Medicine, 2016(4): 50-52.

[15] Zhang Yongjie, Song Changming, Feng Ying. (2011). Clinical Observation on 29 Cases of Advanced Non-small Cell Lung Cancer Treated by Warm-yang Method and Chemotherapy [J]. Shandong Journal of Traditional Chinese Medicine, 30(10): 722-723.

[16] Zhu Wei Ju, Chen Su Sheng. (2011). Cold injury is difficult [M]. Zhang Xiaoxia, school. Beijing: Xueyuan Publishing House, 2011: 114.

[17] Lu Hai Rui, Chen Xinyi. (2014). Application of Ointment in Cancer Therapy and Experience of Professor Chen Xinyi’s Application of Ointment [D]. Beijing: Beijing University of Traditional Chinese Medicine, 2014: 46-48. 